\title{
Upcoming Meetings Related to Alzheimer's Disease
}

\author{
MARCH 2015 \\ Conference: $\quad 9$ th Annual Drug Discovery For Neurodegeneration Conference \\ Date: 1-3 March 2015 \\ Location: $\quad$ San Diego, CA, USA \\ URL: $\quad$ http://www.worldeventsforum.com/addf/drugdiscovery/index.html \\ Conference: $\quad 12$ Annual World Congress of the Society for Brain Mapping \& Therapeutics \\ Date: 6-8 March 2015 \\ Location: $\quad$ Los Angeles, CA, USA \\ URL: $\quad$ http://www.worldbrainmapping.org/general \\ Conference: $\quad$ Keystone Symposia on Molecular and Cellular Biology: Biology of Sirtuins \\ Date: $\quad$ 8-12 March 2015 \\ Location: $\quad$ Santa Fe, NM, USA \\ URL: $\quad$ https://www.keystonesymposia.org/index.cfm?e=web.Meeting.Program\&meetingid=1324 \\ Conference: $\quad$ Alzheimer's Research UK Conference 2015 \\ Date: $\quad$ 10-11 March 2015 \\ Location: $\quad$ London, UK \\ URL: http://www.alzheimersresearchuk.org/annual-conference-2015/ \\ Conference: $\quad$ AD/PD ${ }^{\mathrm{TM}}$ 2015: The 12th International Conference on Alzheimer's and Parkinson's Diseases \\ Date: $\quad$ 18-22 March 2015 \\ Location: $\quad$ Nice, France \\ URL: $\quad$ http://www2.kenes.com/adpd/Pages/Home.aspx \\ Conference: $\quad 9$ th World Congress on Controversies in Neurology (CONy) \\ Date: 26-29 March 2015 \\ Location: Budapest, Hungary \\ URL: $\quad$ http://www.comtecmed.com/cony/2015/Default.aspx \\ APRIL 2015 \\ Conference: \\ Date: \\ 17th International Neuroscience Winter Conference \\ Location: $\quad$ Sölden, Austria \\ URL: http://www.winterneuroscience.org/2015/ \\ Conference: $\quad 30$ th International Conference of Alzheimer's Disease International (ADI) \\ Date: 15-18 April 2015 \\ Location: $\quad$ Perth, Western Australia, Australia \\ URL: $\quad$ http://www.adi2015.org/
}




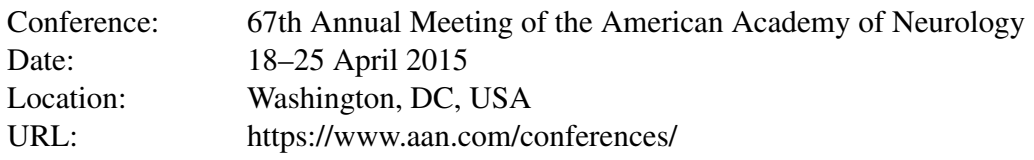

MAY 2015

Conference:

Date:

44th Annual Meeting of the American Aging Association

URL:

Marina del Rey, CA, USA

http://www.americanagingassociation.org/meeting

JUNE 2015

Conference:

Date:

Location:

URL:

Conference:

Date:

Location:

URL:

Conference:

Date:

Location:

URL:

Conference:

Date:

Location:

URL:

Conference:

Date:

Location:

URL:

Conference:

Date:

Location:

URL:

\section{JULY 2015}

Conference:

Date:

Location:

URL:

\section{AUGUST 2015}

Conference:

Date:

Location:

URL:
2015 Annual Meeting of the American Association of Neuropathologists

11-14 June 2015

Denver, CO, USA

http://www.neuropath.org/meetings/

Mechanisms of Neurodegeneration

14-17 June 2015

Heidelberg, Germany

http://www.embo-embl-symposia.org/symposia/2015/EES15-03/index.html

19th International Congress of Parkinson's Disease and Movement Disorders

14-18 June 2015

San Diego, CA, USA

http://www.mdscongress2015.org/Congress-2015.htm

1st Congress of the European Academy of Neurology

20-23 June 2015

Berlin, Germany

http://www.eaneurology.org/berlin2015/

2015 Alzheimer's Disease Congress

23-25 June 2015

London, UK

https://www.regonline.co.uk/builder/site/Default.aspx?EventID=1563612

XXVIIth International Symposium on Cerebral Blood Flow, Metabolism and Function \& XIIth International Conference on Quantification of Brain Function with PET

27-30 June 2015

Vancouver, Canada

http://brain.kenes.com/

Alzheimer's Association International Conference

18-23 July 2015

Washington, DC, USA

http://www.alz.org/aaic/

International Conference on Parkinson's Disease and Movement Disorder

11-13 August 2015

Frankfurt, Germany

$\mathrm{http}: / /$ parkinsons.conferenceseries.com/ 
Conference:

Date:

Location:

URL:

Conference:

Date:

Location:

URL:

Conference:

Date:

Location:

URL:

\section{OCTOBER 2015}

Conference:

Date:

Location:

URL:

\section{Conference:}

Date:

Location:

URL:

Conference:

Date:

Location:

URL:

Conference:

Date:

Location:

URL:

\section{MARCH 2016}

Conference:

Date:

Location:

URL:

\section{APRIL 2016}

Conference:

Date:

Location:

URL:

\section{SEPTEMBER 2016}

Conference:

Date:

Location:

URL: 25th Meeting of the International Society for Neurochemistry

23-27 August 2015

Cairns, Australia

http://www.neurochemistry.org/biennial-meeting/isn-2015-biennial-meeting.html

2nd International Conference on Geriatrics \& Gerontology

24-26 August 2015

Toronto, Canada

http://geriatrics-gerontology.conferenceseries.com/

3rd International Conference on Alzheimer's Disease and Dementia

31 August - 2 September 2015

Toronto, Canada

http://alzheimers-dementia.conferenceseries.com/

$8_{\text {th }}$ Canadian Conference on Dementia

1-3 October 2015

Ottawa, Canada

http://www.canadianconferenceondementia.com/

8th Iberoamerican Congress on Alzheimer's Disease

15-17 October 2015

Rio de Janeiro, Brazil

http://www.aibbrasil2015.com.br/ingles/index.php

SfN: Neuroscience 2015

17-21 October 2015

Chicago, IL, USA

http://www.sfn.org/annual-meeting/neuroscience-2015

XXII World Congress of Neurology - WCN 2015

31 October -5 November 2015

Santiago, Chile

http://www.wen-neurology.com/

14th International Athens/Springfield Symposium on Advances in Alzheimer Therapy 9-12 March 2016

Athens, Greece

http://www.ad-springfield.com/

31st International Conference of Alzheimer's Disease International (ADI)

20-24 April 2016

Budapest, Hungary

http://www.alzint.org/2016

4th World Parkinson Congress (WPC 2016)

20-23 September 2016

Portland, OR, USA

http://www.worldpdcoalition.org/?page=WPC2016 